'Departamento de Hematología y Oncología, Pontificia Universidad Católica de Chile. Santiago, Chile.

${ }^{2}$ Departamento de Medicina

Interna, Pontificia Universidad Católica de Chile. Santiago, Chile.

Recibido el 20 de mayo de 2016, aceptado el 9 de mayo de 2017.

Correspondencia a:

Dr. Mauricio Sarmiento Lira 85 4to piso. Santiago, Chile. mauriciosarmiento@hotmail.com

\section{Leucemia linfoblástica aguda con grave alteración hepática como manifestación inicial. Presentación de dos casos y revisión de la literatura}

\author{
MURIEL CATALÁ², MAURICIO OCQUETEAU T. ${ }^{1}$, \\ MAURICIO SARMIENTO M. ${ }^{1}$
}

\section{Acute lymphoblastic leukemia causing hepatic failure. Report of two cases}

\begin{abstract}
Hematological diseases may cause acute hepatic failure and are seldom sought. We report two cases illustrating this situation. A 16-year-old male presented with an acute liver failure: a bone marrow biopsy showed complete infiltration with lymphoid type blasts. A flow cytometry confirmed the presence of an acute lymphoblastic leukemia. The patient died days later. A 20-year-old female presented with acute liver failure: due to a pancytopenia, a bone marrow biopsy was performed, which confirmed the presence of an acute lymphoblastic leukemia. Chemotherapy was started immediately. The patient had a favorable evolution.

(Rev Med Chile 2017; 145: 804-807)

Key words: Acute Hepatic Failure; Acute Lymphoblastic Leukemia; Flow Cytometry.
\end{abstract}

$\mathrm{E}^{\prime}$ 1 compromiso hepático secundario en las enfermedades hematológicas es un hallazgo frecuente y generalmente se manifiesta como lesión focal o leves alteraciones en la bioquímica hepática. Suelen presentarse en fases avanzadas de linfoma no Hodgkin ${ }^{1}$, leucemia linfática crónica $^{2}$ y enfermedad de Hodgkin ${ }^{3}$, entre otras. Sin embargo, el compromiso grave de la función hepática es infrecuente, con desalentadores resultados, en los cuales el diagnóstico suele ser post mortem $^{4-6}$. Algunos de los casos en leucemias agudas obedecen a reactivación de virus de la familia herpes, en contexto de la inmunosupresión generada por la quimioterapia ${ }^{7}$. El compromiso primario derivado de la leucemia es infrecuente. Este reporte describe los casos de 2 pacientes con leucemia linfoblástica aguda y graves alteraciones de la función hepática como manifestación inicial, con desenlaces opuestamente diferentes.

\section{Casos clínicos}

\section{Caso 1}

Paciente varón de 16 años, sin antecedentes mórbidos de importancia, inició su enfermedad un mes previo a la hospitalización con epistaxis a repetición y hemorragia gingival. No hubo atención médica en estos eventos. Una semana antes del ingreso consultó por odinofagia y fiebre $\mathrm{y}$, bajo sospecha de amigdalitis aguda bacteriana, se prescribió penicilina benzatina. Dos días previos al ingreso presentó ictericia progresiva y compromiso de consciencia. Ingresó al hospital público cercano a su domicilio confuso y con ictericia intensa. En ese momento el laboratorio mostró: Tiempo de protrombina (TP) 100 segundos, INR: 6,5, tiempo de tromboplastina parcial activada (TTPA) 37,5 segundos, SGOT (transaminasa glutámico oxaloacética sérica) $12.000 \mathrm{UI} / \mathrm{L}$, 
SGPT (transaminasa glutámico pirúvica sérica) $10.560 \mathrm{UI} / \mathrm{L}, \mathrm{GGT}$ (gamma glutamil transpeptidasa) $87 \mathrm{UI} / \mathrm{L}$, bilirrubina total (BT) 18,6 mg\% y directa (BD) $17,1 \mathrm{mg} \%$, creatinina (Cr) $1,1 \mathrm{mg} \%$, glóbulos blancos (GB) 1.500/uL, hemoglobina (Hb) $9 \mathrm{~g} \%$, plaquetas (PLT) $15.000 / \mathrm{uL}$.

Ante la sospecha de hepatitis fulminante, fue derivado a nuestro hospital, para trasplante hepático prioritario. Ingresó grave, con inestabilidad hemodinámica y encefalopatía de difícil manejo, contraindicándose monitoreo invasivo de la presión intracerebral por pancitopenia grave: PLT 10.000/ uL, GB 800/uL, Hb7g\%, y falla hepática con fibrinógeno 176 mg\%, SGOT 2.940 UI/L, SGPT 2.037 UI/L, GGT $48 \mathrm{UI} / \mathrm{L}$, ácido láctico $8,6 \mathrm{mmol} / \mathrm{L}$, BT 16,2 mg\%, BD 8 mg\%, INR 2,2 (post soporte transfusional), inmunoglobulinas en rangos normales y LDH 9.000 UI/L. Fue evaluado por equipo de trasplante hepático y se complementó estudio con niveles de acetaminofeno que resultaron en 6,0 $\mathrm{ug} / \mathrm{mL}$ (valor normal en hasta $20 \mathrm{ug} / \mathrm{mL}$ ), ferritina $50.000 \mathrm{U} / \mathrm{mL}$ (rango normal 20-200 U/mL), anticuerpos anti LKM1 4,2 U/mL (rango normal de 0 a 18), anticuerpos antinucleares negativos, anticuerpos antimúsculo liso negativos y anticuerpos antimitocondriales negativos. Se activó el programa nacional de donantes ubicándolo primero en la lista de espera. Fue evaluado por infectología y se realizaron diversos estudios, entre los que se encontraron: hemocultivos negativos, anticuerpos VIH negativos, RPR no reactivo, antígeno de superficie de hepatitis $\mathrm{B}$ negativo, anticuerpos virus hepatitis $\mathrm{C}$ negativo, IgM citomegalovirus negativo e IgG positivo, anticuerpos para tripanosoma cruzi negativos, IgG parvovirus positivo, IgM parvovirus negativo, IgG varicela zoster negativo y anticuerpos anti leptospira negativos. Fue evaluado por el equipo de hematología, realizando mielograma que mostró infiltración total por blastos de estirpe linfoide y la citometría de flujo confirmó leucemia linfoblástica aguda estirpe B, CD10+, CD20+. Se inició profilaxis de lisis tumoral con volemización, alopurinol, alcalinización urinaria y metilprednisolona $40 \mathrm{mg}$ endovenosos cada $12 \mathrm{~h}$. La evolución clínica fue hacia el deterioro neurológico y hemodinámico y con elevación progresiva de la creatinina y productos nitrogenados, requiriendo terapia de reemplazo renal. Hubo agravamiento de todos los parámetros antes mencionados y el paciente falleció a causa de shock hemorrágico por sangrado digestivo y pulmonar.

\section{Caso 2}

Pacinte de sexo femenino de 20 años, sin antecedentes relevantes, consultó por historia de 2 meses de infecciones respiratorias, diaforesis nocturna y sensación febril no cuantificada, a lo cual se había agregado vómitos e ictericia. Al examen de ingreso se encontraba ictérica, con petequias y palidez. Se realizaron exámenes donde se encontró pancitopenia, con GB 1.700/uL, Hb 9 g\%, PLT 14.000/uL y bioquímica alterada con BT 10 mg\%, BD 7,8 mg\%, FA 242 UI/L SGOT 2.040 UI/L SGPT 1.927 UI/L BUN 8,8 mg\%, LDH 504 UI/L, PCR 6,2 mg\%. En contexto de estudio de hepatitis se evaluó: serología de virus hepatitis $A, B$, C negativos; VIH negativos, ANA (+) 1/1.280, anti DNA positivo, complemento normal y perfil ENA positivo. Fue evaluada por Reumatología que no encontró hepatitis autoinmune categórica. El siguiente control de exámenes mostró TP 48\%, INR 1,5, TTPA 37,5 s, SGOT 12.900 UI/L, SGPT 10.550 UI/L, GGT 89 U/L, BT 18,6 mg\%, BD 17,1 mg\%, Cr 1,1 mg\%, GB 840/uL, Hb 5,8 g\%, PLT 13.000/ uL. Fue evaluada por Hematología para establecer si la pancitopenia era secundaria a enfermedad reumatológica o enfermedad medular primaria. Se realizó mielograma y citometría de flujo, los cuales mostraron presencia de LLA CALLA $(+)$, con expresión parcial de CD20 y $(9 ; 22)$ negativa. De forma inmediata se inició tratamiento con esquema quimioterapia BFM con dexametasona a dosis de $20 \mathrm{mg} / \mathrm{m}^{2}$ día, con lo que se observó normalización de las pruebas hepáticas (Tabla 1). La evolución de la paciente ha sido favorable, con normalización de pruebas hepáticas posterior al inicio de la quimioterapia, y logrando, al término del primer ciclo de quimioterapia, remisión completa de su leucemia.

\section{Discusión}

La insuficiencia hepática aguda resulta de una gran variedad de causas (Tabla 2). La frecuencia de las diferentes causas varía en las diferentes regiones. Para Chile, las principales causas son las infecciones virales e intoxicaciones ${ }^{8,9}$.

El desarrollo de falla hepática fulminante puede llegar a gravedad extrema que requiera la realización de un trasplante hepático ${ }^{9}$. Entre las condiciones que contraindican el trasplante se encuentran: falla orgánica múltiple, infección no 
Tabla 1. Evolución cronológica de exámenes de laboratorio

\begin{tabular}{|lccccccc|}
\hline & Día 1 & $\begin{array}{c}\text { Paciente 1 } \\
\text { Día 4 }\end{array}$ & Día 6 & Día 1 & Páa 6 & Día 13 & Día 23 \\
\hline Hb & 9 & 7 & 4 & 9 & 8 & 9 & 10 \\
\hline GB & 1.500 & 800 & 500 & 1.700 & 840 & 0 & 5.600 \\
\hline PLT & 15.000 & 10.000 & 2.000 & 14.000 & 15.000 & 4.000 & 96.000 \\
Cr & 1,1 & 4 & 6 & 0,8 & & 0,6 & 0,5 \\
\hline BT & 18,6 & 16,2 & 22 & 10 & 18,6 & 3,97 & 2,13 \\
\hline BD & 17,1 & 8 & 20 & 7,8 & 17,1 & 1 & 0,8 \\
\hline INR & 6,5 & 2,2 & 6 & 1,6 & 1 & 1 & 1 \\
\hline FA & 400 & 300 & 248 & 242 & & 321 & 291 \\
\hline SGOT & 12.000 & 2.940 & 22.000 & 2.040 & 12.900 & 142 & 72 \\
\hline SGPT & 10.560 & 2.037 & 21.000 & 1.927 & 10.550 & 301 & 205 \\
\hline GGT & 87 & 48 & 49 & & 89 & 80 & 40 \\
\hline LDH & 9.000 & & 12.000 & 504 & & & \\
\hline
\end{tabular}

Tabla 2. Causas de falla hepática fulminante

Hepatitis viral
Virus de hepatitis A, B, C, D, E, herpes simplex, Epstein Barr, citomegalovirus, herpes virus 6, influenza B, virus de fiebre
hemorrágica (Lassa, Ebola, Marburg)
Drogas
Paracetamol (accidental o intento suicida), antibióticos (isoniazida, nitrofurantoína, tetraciclina, ciprofloxacino, eritromicina,
amoxicilina-ácido clavulánico), ácido valproico, lovastatina, fenitoína, antidepresivos tricíclcios, halotano, oro, flutamida,
antabuse, ciclofosfamida, loratadina, propiltiouracilo, diclofenaco, sulfas, amiodarona, entre otras
Toxinas
Amanita Phalloides, solventes orgánicos, hierbas medicinales (ginseng, valeriana, té rojo, etc), toxinas bacterianas (Bacillus
Cereus, cianobacterias)
Misceláneas
Hígado graso agudo en embarazo, síndrome HELLP, hepatitis autoinmune, Budd-Chiari, trombosis portal, insuficiencia
cardiaca derecha, hepatitis isquémica, leucemia, linfoma, metástasis, malaria, tuberculosis, síndrome de Reye, enfermedad
de Wilson
Indeterminadas

controlada, daño cerebral irreversible y neoplasia extrahepática. En esta última categoría cabe la leucemia aguda. La fisiopatología de la falla hepática en neoplasias hematológicas se asocia a infiltración blástica perisinusoidal, con patrones bioquímicos similares a los observados en la hepatitis isquémica ${ }^{7}$.

En la literatura, el origen hematológico maligno de la falla hepática ha sido escasamente publicado. Por esta misma razón, no hay grandes series de enfermos que permitan tener la suficiente experiencia para asegurar un adecuado tratamiento. Entre los primeros reportes encontrados se describe 24 enfermos, principalmente afectados por linfoma, de los cuales sólo sobrevivieron 3 en un período breve de observación ${ }^{11}$. La alta mortalidad de la falla hepática asociada a neoplasia hematológica ha sido reportada. Litten et $\mathrm{al}^{12}$, describen el caso de un paciente de 4 años que inició un cuadro súbito de dolor abdominal e ictericia con 
un rápido curso de falla hepática. Los exámenes de laboratorio mostraron hepatitis aguda de causa no precisada y no había mayores alteraciones hematológicas salvo leve trombopenia. Se realizó trasplante urgente y la biopsia rápida intraoperatoria confirmó compromiso por leucemia o linfoma agudo. El trasplante hepático fue suspendido y luego de $12 \mathrm{~h}$ de estancia en la Unidad de Cuidados Intensivos el paciente falleció. Entre las principales dificultades para tratar estos enfermos se encuentran el peligro de realizar una biopsia hepática y la elección de la quimioterapia según la potencial toxicidad hepática. Entre los pocos casos reportados que han sobrevivido se han asociado el tratamiento precoz con corticoides y el uso de etoposido, fármaco de elección, con menor tasa de metabolismo hepático ${ }^{12}$.

Nuestros casos muestran las grandes dificultades en el diagnóstico de esta situación. El primer caso tuvo una brusca y rápidamente progresiva evolución y el paciente falleció sin haber recibido quimioterapia alguna. En nuestro servicio se adoptó una estrategia de alerta ante casos de falla hepática de causa incierta con alteración al hemograma. Años después tuvimos la oportunidad de tratar a la segunda paciente en quien se realizó un diagnóstico precoz y que, afortunadamente, luego del tratamiento con quimioterapia, logró sobrevivir a esta grave asociación entre leucemia aguda y falla hepática. Si bien en este caso podría plantearse que la alteración hepática estaba asociada a enfermedad inmune, la dramática respuesta al tratamiento con agentes antineoplásicos sugiere la asociación con la leucemia. Para poder discernir, esto sería ideal contar con una biopsia hepática, pero en nuestros pacientes la biopsia por punción o la biopsia por vía transyugular no fueron realizadas por la gran coagulopatía y las intensas citopenias.

\section{Conclusión}

La falla hepática asociada a neoplasia hematológica es una condición infrecuente, pero de alta mortalidad. Compartir nuestras experiencias busca sensibilizar a los equipos de trasplante hepático, intensivistas y gastroenterólogos a pensar en el concurso rutinario del hematólogo en los casos donde la causa no sea evidente y en casos en que se encuentre hemograma alterado no explicado por la enfermedad de base o complicaciones (neutropenia, trombopenia profunda, leucopenia, etc.).

\section{Referencias}

1. Salmon JS, Thompson MA, Arildsen RC, Greer JP. Non Hodgkin's Lymphoma involving the liver: clinical and therapeutic considerations. Clin Lymphoma Myeloma 2006; 6 (4): 273-80.

2. Shebab T, Kaminski M, Lok A. Acute liver failure due to hepatic involvement by hematologic malignancy. Digestive diseases and sciences, 1997; 42 (7): 1400-5.

3. Lefkowith JH, Falkow S, Whitlock RT. Hepatic Hodgkin's disease simulating cholestasic hepatitis with liver failure. Arch Pathol Lab Med 1985; 109: 424-6.

4. Anderson S, Richardson P, Wendon J, Pagliuca A, Portmann B. Acute liver failure as the intial manifestation of acute leukemia. Liver 2001; 21: 287-92.

5. Matsuzaki A, Suminoe A, Koga Y, Kusuhara K, Hara $\mathrm{T}$, Ogata $\mathrm{R}$, et al. Fatal visceral varicella-zoster virus infection without skin involvement in a child with acute lymphoblastic leukemia. Pediatr Hematol Oncol 2008; 25 (3): 237-42.

6. Suso A, Sola A, Osay L, Moreno A, Salvatore A, Carena J. Hepatitis fulminante fatal como presentación de una leucemia linfática aguda. Medicina (Buenos Aires) 2014; 74: 177-9.

7. Reddi DM, Barbas AS, Castleberry AW, Rege AS, Vikraman DS, Brennan TV, et al. Liver transplantation in an adolescent with acute liver failure from acute lymphoblastic leukemia. Pediatric Transplantation 2014: 18: E57-63.

8. Clocca M, Moreira-Silva SF, Alegria S, Galoppo MC, Ruttiman R, Porta G, et al. Hepatitis A as an etiologic agent of acute liver failure in Latin America. Pediatr Infect Dis J 2007; 26 (8): 711-5.

9. Uribe M, Buckel F, Ferrario M, Godoy J, Blanco A, Hunter B et al. Epidemiology and results of liver transplantation for acute liver failure in Chile. Transplant Proc 2003; 35 (7): 2511-2.

10. Wolff GM, Petrovic LM, Rojter SE, Villamil FG, Makowka L, Podesta LG, et al. Acute liver Failure due to lymphoma. Dig Dis Sci 1994; 39 (6): 1351-8.

11. Shehab TM, Kaminski MS, Lok AS. Acute liver failure due to hepatic involvement by hematologic malignancy. Dig Dis Sci 1997; 42 (7): 1400-5.

12. Litten J, Rodríguez M, Maniaci V. Acute lymphoblastic leucemia presenting in fluminant hepatic failure. Pediatr Blood Cancer 2006; 47: 842-5. 